\title{
Mobile Internet Preference Recommendation Based on Scene Model
}

\author{
Ren Yongguo, Pang Long
}

\author{
Communication University of China, Network Engineering Department, Beijing100024, China
}

Keywords: music recommendation; scene model; user preferences; mobile client

\begin{abstract}
The current domestic online music providers, such as QQ music, KuGou music, Baidu Music, etc., most of which support keywords retrieval of users to search songs, listen online, download songs, and other functions. Few manufacturers provides functions as 'songs may conform to your taste', 'songs of similar style' etc. However, there is still a more complex operating path for the users to truly find their favorite songs and start listening. In order to solve this problem, we put forward the solution recommended with personalized music scene, enabling the users to play their favorite music at proper time, proper place quickly and freely without too much knowledge of the music and a large amount of energy.
\end{abstract}

\section{Introduction}

In the forefront of the researches of digital music, there is an urgent demand on improving music retrieval efficiency and personalized recommendation of music. Merely by putting up the 'artist', 'title' and 'genre' symbols is hard to help users find their favorite music. This old method is achieved by text information and the editable metadata, or the existing data of purchasing behavior (since you bought this one, you may also like the one of the same kind). And the new method uses a large potential database in which the users' interests were recorded, and extracts the semantic description of music files, or collects tagging information from the user community, and combines all these information to recommend personalized music.

\section{The Environment Modeling and Recommended Situational Awareness of Mobile Platform}

We need to establish more accurately user scene mode. Besides, we need to collect enough information of user's scene model, and give different weights to users' characteristics under different dimensions. Finally, we can couple with a variety of recommendation algorithm to recommend corresponding music to the user.

First of all, in recent years, the user's location information acquisition, with the popularity of smart phones, is becoming more and more convenient. As long as we apply appropriate permissions and privacy protection, by cellular phone base stations, IP address and wifi hot pints and GPS information, we can accurately infer the user's location information. Beside, the location of the user is directly related to the user's mood and the kind of music he want to listen to. For example, the music the user wants to listen while he is working overtime in his office may be utterly different from the one while he is resting at home.

User's time information is also very important. If the user's life time arrangement is regular, then time information is very important information source. For the users who get up early every day to do exercise, they may need to listen to some music to cheer up. Some users may stay up very late at night, for them, some quiet lullabies may be proper.

Some information of listening habits, as the weather conditions, social events, fashion and so on that may influence the users' choosing of songs is also very important. Every year on April 1, Leslie Cheung's anniversary, for example, songs sung by him are more frequently played that average quantity. 
On the recommended algorithm, first of all, we need a mechanism to grade the users' songs, in simple terms, for example, for a certain piece of song, if users collect or download this piece of song, the mechanism should rise the score. If the User deleted this song, the grade should be reduced. From the above, we can summarize the relation matrix of the grades between the user and song items. This is a typical collaborative filtering algorithm.

Guess the score of
item 1 for user5?
\begin{tabular}{|c|c|c|c|c|c|c|c|c|c|c|c|c|}
\hline & 1 & 2 & 3 & 4 & 5 & 6 & 7 & 8 & 9 & 10 & 11 & 12 \\
\hline 1 & 1 & & 3 & & $?$ & 5 & & & 5 & & 4 & \\
\hline 2 & & & 5 & 4 & & & 4 & & & 2 & 1 & 3 \\
\hline 3 & 2 & 4 & & 1 & 2 & & 3 & & 4 & 3 & 5 & \\
\hline \multirow{3}{*}{$*$} & & 2 & 4 & & 5 & & & 4 & & & 2 & \\
\hline 5 & & & 4 & 3 & 4 & 2 & & & & & 2 & 5 \\
\hline 6 & 1 & & 3 & & 3 & & & 2 & & & 4 & \\
\hline
\end{tabular}

As shown in the above figure, the recommended work automatically generates as correction of a certain piece of song that the user never played too much. We can get the result simply apply the collaborative filtering method.

\section{Personalized Designed Recommended Architecture of Scene Music}

Personalized recommended architecture of scene music contains two parts, namely, the perception system of the scene and personalized recommendation system. Combining these two parts, a personalized song list could be generated with the music scene.

3.1 Scene Perception System Design

As stated earlier, the information obtained through mobile sensors and client applications would be sent to the back-end log platform in real-time or delayed. The back-end cloud computing platform would analyze and process the log information of the users in every day, and find the scenes that the user in of each period, and then generate a list of recommended scenario of each time and each place.

The collection of users' characteristics is mainly performed by mobile client. Through the customers' permission, we collect the users' music history and history retrieval and other operations, and the users' login account information, and the information te users left in other product lines, etc. Such as through BBS posts, we could get the user information and unify the information to cloud storage space, and then through recommended offline cloud computing service there would generate corrected user label and recommended song list every day.

3.2 System Design of Personalized Recommendation

Whether the recommendation function can achieve the desired effect mostly lie in constant triggering and feedback of the users' participation, and letting the users to correct our recommended results, at the same time, helping us to improve our recommendation algorithm. Different users' feedbacks (such as switching songs or collecting, deleting songs etc.) will give different weights correspondingly, thus affect the recommendation results. 


\subsection{Scene Music Design of Personalized Mobile Platform}

The recommendation of the mobile platform itself bears the following differences compared with the traditional ones.

(1) Users on mobile platforms can use the client end to access the recommendation system at any time and in any location. Results recommended by mobile users should be closely related with the situational information, such as the time and location. The mobile recommendation of situational awareness requires stricter real-time demand than that of the traditional ones.

(2) There is discreteness of the recommendation of the mobile platform. In many cases, there is no or weak network connection in mobile platform or. Therefore, we need to consider the problem of calculation offline or calculate lightly rely on network.

(3) The mobile devices are of small screens, so the display space is limited, and it is inconvenient for us to operate. Therefore, the recommendations should be made to adapt to corresponding changes. For example, the recommended results should be concisely showed to consumers, in this way to save the operation of clicking the small button. In view of the music application, we can even consider the form of inserting audio to show our recommendation result. Such as inserting some voice hints messages between two songs to attract users pay attention to our recommendation results.

For the scene needs recommended by mobile platform, we designed the personalized playlist with scenario list. And the specific design diagram is as the following:

Compared with the previous mentioned separate scene recommendation, this process combines with the users' personality, thus it provides finally a list of the scene playlists which may conform to the current state of the users. Besides, each scene playlist contains a personalized list of songs containing the personal information and habits of the users.

\section{Specific Implementation of Personalized Recommended Scene Songs}

Situational awareness is not achieved overnight. In order to accurately distinguish the situational awareness as soon as possible, we need a long time of searching of data and deciding based on the analysis of characteristics of the users. At the same time, we also need take the users' actively participation degree into consideration to choose to optimized algorithm. Next, through concrete instance analyses, we illustrate how identification and distinction is done in specific situations.

4.1 Scene Recognition of Fixed Position and Fixed Time

Taking the users' identity label information into consideration is a continuous loop optimization process while we making the scene music recommendation in a particular period of time.

This process, first, requires a huge music library that has already been classified. Based on the original attribute tags of the music, we can put most of the songs in certain scene classification artificially. And then, according to the users' "listening" and "slice" operations to check whether these songs are classified accurately.

\subsection{Scene Recommendation Notifications at Specific Situation}

In order to improve the users' active degree and speed up the optimization of our recommendation algorithm, at the right time, we need to remind the users to listen to our scenario music, or when users forget this function, we can timely delivery to the users the music they want to listen to. Because only by the constantly listening and operation of the users, can we recommend more accurate songs. In this way we can make the recommended songs both are new and the type the users likes, at the same time, the songs we recommended are in accordance with his state of mind at that time. We call the whole function personalized scene songs recommendation function.

The core of this design lies in finding out the optimization process of the algorithm and the precision requirements of the recommendation. Through finding out a large number of data 
algorithm method, we can dig up the users that need to be timely recommendation and the corresponding by suitable to the user and need time to recommendation the recommendation playlist. And then, in every morning this mechanism would recommend service, and use cloud computing to generate the list of users and recommendation result, and then use the API of the recommendation system to generate a set of tasks. Timing task, the next day, will send the recommendation list to the user's mobile device instantly when it is right.

If there is a piece of big news of the day, we should recommend it through the broadcast as a recommendation task to all users. In this condition, we can cancel all the preset timing recommendation tasks. And in this way, we can avoid setting too many recommendations to bother the users.

\subsection{Timely Insert Audio to Present Voice Recommendation}

Due to the facts that the screen of mobile platform is small, the operation is not convenient, for a music application, though it is likely to occupy the users a long period of time, in fact while listening to music the user basically won't stare at the screen. So how can we make the recommendation results with timeliness to be truly noticed by users? We think of inserting voice prompt between two songs, which is similar to the traditional digital FM radio music, in which the host can insert his own comments or recommendations even commercial advertising between the intervals. In fact, this is why this scheme catches attention of the industry. There is little profit of the present audio applications. It should be paid much attention if there is the possibility for us to insert audio advertising between intervals. Then we take the advertising as an example to describe the design scheme of audio recommendation, SDK refers to the code library of the inserted audio in the mobile client end, including audio broadcast core and network module through which we could access to audio resources.

The application scenario of part is very rich. For example, if you are sitting and listening to music to rest in a cafe of a mall, at this time, the mall you are in is holding a small musician meeting, and this musician is the one you like. However, you just don't know this piece of information. It is a pity if you have missed it. In this case, our audio recommendation ads will be of great use. At the intervals of your listening to music, the prompt voice broadcast speech would remind you of the small meeting. Therefore, our system meets the very important requirements of user.

\section{Conclusion}

Based on the above theoretical description of the basic structure of scene perception system and the basic structure and process of personalized recommendation, take the two designs into consideration, we finally elaborated the carrying out personalized scene music on the mobile platform and its final implementation scheme. At the same time, we also discussed some problems we encountered in the implementation stage, and put forward the optimization solution. Finally, we introduced the voice recommendation scheme and the design scheme of advertising recommendation. Actually,the goals of all our recommendations or scenarios lie in meeting the needs of users and improve the viscosity of our service.Most importantly, all the above aims at make the business commendation ads known to the users. And in this paper, we also talked about the music client design and implementation of mobile phone under mobile environment, as well as the potential problems.

\section{References}

[1] F. Pachet and J.J. Aucouturier. Improving Timbre Similarity: How High is the Sky? Journal of negative results in speech and audio sciences, 1(1):1-13, 2004. 
[2] Bass Lines, Emiru Tsunoo, George Tzanetakis, and Nobutaka Ono. Beyond Timbral Statistics : Improving Music Classification Using Percussive. IEEE Transactions on Audio, Speech and Language Processing, 19(4):1003-1014, 2011.

[3] George Tzanetakis, Student Member, and Perry Cook. Musical Genre Classification of Audio Signals. IEEE Transactions on Speech and Audio Processing,10(5):293-302, 2002.

[4] M Mandel. Song-level Features and Support Vector Machines for Music Classification. In Proc. International Conference on Music, 2005.

[5] Janet Marques and Pedro J Moreno. A Study of Musical Instrument Classification Using Gaussian Mixture Models and Support Vector Machines, 1999.

[6] Alexandra Uitdenbogerd and van Schyndel Ron. A Review of Factors Affecting Music Recommender. In 3rd International Conference on Music Information Retrieval (2002), 2002. 